\title{
The Long-Term Effects of Early Kwashiorkor Compared with Marasmus. IV. Performance on the National High School Entrance Examination
}

\author{
JANINA R. GALLER, FRANK C. RAMSEY, DEBRA S. MORLEY, ESTHER ARCHER, AND \\ PATRICIA SALT \\ Center for Behavioral Development and Mental Retardation, Boston University School of Medicine, \\ Boston, Massachusetts 02118 and Department of Pediatrics, Queen Elizabeth Hospital, Bridgetown, Barbados
}

\begin{abstract}
This is one of a series of studies on the longterm effects of early childhood malnutrition in Barbadian school children. This is the first report of the relationship between early malnutrition and later performance on a national examination administered to all 11-y-old children in Barbados to assign high school seats. We compared scores achieved on the 11-plus examination by 103 boys and girls with histories of marasmus or kwashiorkor with those obtained by 63 healthy comparison children and also with scores obtained by the total island population of children during the same years. We report that children with histories of either type of malnutrition confined to infancy had significantly lower scores on the national high school examination than healthy comparison children. Reduced 11-plus scores were closely associated with teacher reports of attention deficits in the classroom documented when the children were as young as 5 to $8 \mathrm{y}$ of age and also with IQ and academic performance. Early malnutrition had independent effects on performance on the 11-plus examination even when home environmental conditions were controlled for. These findings have important implications for future opportunities available to children with histories of infantile malnutrition. (Pediatr Res 28: 235239, 1990)
\end{abstract}

\section{Abbreviations}

IQ, intelligence quotient

ANOVA, analysis of variance

MRA, multiple regression analysis

In a series of studies in Barbados (1-3), we have followed the growth and behavioral development up to 18 y of age of boys and girls with histories of marasmus and kwashiorkor in the first year of life and have compared the findings with those obtained from healthy Barbadian control children matched by age, sex, and socioeconomic group. There was no evidence of continuing malnutrition in this group and follow-up after puberty of boys and girls in this category demonstrated catch-up growth that gave them the same body dimensions as the control children (1).

Received January 25, 1990; accepted April 23, 1990.

Reprint requests: Dr. Janina R. Galler, Center for Behavioral Development and Mental Retardation, M921, Boston University School of Medicine, 80 East Concord Street. Boston, MA 02118.

This research was conducted with the cooperation of the Ministries of Health and Education, Barbados, and was supported by grants from the Ford Foundation and The Thrasher Research Fund. F.C.R. was supported by the Milbank Fund and the W.T. Grant Foundation. This research was approved by the Boston University School of Medicine Institutional Review Board for Human Subjects.
However, the previously malnourished children had continuing deficits in cognitive and behavioral functioning through the school years, which included impaired IQ performance (2) and attention deficits reported by teachers and parents (4). Socioeconomic and home environmental factors during childhood and adolescence did not play a major role in these findings.

The opportunity presented itself to use the results of the 11plus examination administered to all 11-y-old children in Barbados. Scores achieved on this national examination were used by the Barbadian Ministry of Education to rank children for assignment to local high schools. The highest ranking children were assigned to academic-oriented schools and lower ranking children were, in many cases, assigned to trade schools. Performance on the 11-plus examination therefore was likely to have a major long-term impact on the future careers and opportunities available to the children. To our knowledge, this is the first study relating performance on a national examination to a carefully documented history of infantile malnutrition. This supplements other published reports, including our own, of the association between poor nutrition and educational performance $(5,6)$. We now report that children with histories of marasmus or kwashiorkor confined to infancy had significantly lower scores on the national high school examination than did healthy comparison children. Reduced scores were closely associated with teacher reports of attention deficits in the classroom documented when the children ranged in age from 5 to $11 \mathrm{y}$.

\section{MATERIALS AND METHODS}

Description of study population. This study was undertaken at the National Nutrition Centre, Bridgetown, Barbados, in cooperation with the Ministries of Health and Education of Barbados. From 1967 to 1988, the Centre provided medical care and nutritional surveillance for children with varying degrees of malnutrition from infancy to $11 \mathrm{y}$ of age. Thorough documentation was available on approximately 2100 children with or at risk for malnutrition (7).

Procedures to select participants in this longitudinal study have been described in earlier papers of this series (1-3). Briefly, the children represented nearly all children on the island of Barbados who were born between 1967 and 1972 and who experienced a single episode of malnutrition in their first year of life. All children were examined by one of us (F.C.R.) and admitted to the hospital for a period of approximately $30 \mathrm{~d}$ with a diagnosis of marasmus [moderate to severe weight loss below $75 \%$ of expected weight for age according to the Gomez scale (8) and the absence of edema] or kwashiorkor (characterized by skin changes, edema, and hair changes). Criteria that were satisfied for the current investigation were: 1 ) birth weight $\geq 5 \mathrm{lb}$, excluding cases of fetal growth retardation, 2) absence of any pre-, peri-, or postnatal complications by history, clinical examination, 
and Apgar scores, and 3) absence of encephalopathic events during childhood. After the episode of malnutrition, all children were enrolled through 11 y of age into the National Nutrition Centre, where families were provided with a program of nutrition education, subsidized milk, routine health care, and home visits by a team of community health sisters. Adequate nutrition and good health were assessed by physical examination and red blood cell counts.

Previously malnourished children were matched by sex, age, and handedness (for neurologic tests) with healthy classmates from the same socioeconomic background (comparison group). Three classmates were randomly selected as a possible match for each index child, and final selection was based on access to birth and health records and parental permission. Comparison children met the same selection criteria as the previously malnourished children, but were documented by local health clinics as having adequate growth and nutrition during childhood. Only a few children were excluded on the basis of growth failure; exclusion was generally based on being unable to find complete birth and growth records. Comparison children were in the correct grade for age even when they were matched to index children $(n$ $=37$ ) who were not in the correct grade.

In our study, 39 children with histories of kwashiorkor (a subset of 65 we have followed previously), 64 children with histories of marasmus (a subset of the original sample of 129), and 63 comparison children (also a subset of an original sample of 129) were included. This represented the total number of children for whom the 11-plus scores were available at the time of our survey, conducted when the children were 11 to $18 \mathrm{y}$ old. There were no differences between the participants and children excluded in the current survey with regard to IQ scores or socioeconomic characteristics of the household.

Measures. Evaluations included 11-plus scores, cognitive and behavioral outcomes, and socioeconomic and home characteristics. All testers were blind to the nutritional history of the child.

11-plus examination. Introduced to Barbados in 1959, the 11plus is administered annually to all 11-y-old children in Barbados and, until recently, in all Commonwealth nations, to classify them for the purpose of assigning seats in local secondary schools. The test, which was modified annually, consisted of 140 questions in English and 80 questions in mathematics during the years included in this study. The scores were standardized for age by the Ministry of Health, added, and reported as total 11plus scores. Scores reported in the current study were collected during the period ranging from 1982 to 1984 , when the majority of the children were $11 \mathrm{y}$ of age or older and had completed the examination the same year or up to 7 y earlier. Scores, which were provided by the child's parent or legal guardian, were based on a written report from the Ministry of Education. Because the original report was not always available, 42 scores reported by the parents were cross-referenced with scores obtained directly from the Ministry of Education. The high degree of correlation between the two sets of scores $(r=0.88 ; p<0.001)$ confirmed the reliability of maternal reports. Scores for these 42 cases were not biased in favor of maternal reports.

$I Q$ and classroom behavior. We examined the 11-plus scores in relationship to cognitive and behavioral performance measured when the children were 5 to 11 y of age. The IQ measure was a modified version of the Wechsler Intelligence Scale for Children, which has been described elsewhere (9). To make tests relevant for Barbadian school children, items were altered to include local exemplars. The test was administered by a trained child psychologist. Classroom behavior (10) was reported by teachers who had no knowledge of the nutritional history of the children. A principal components analysis with normal varimax rotation of items in the teacher questionnaire resulted in seven independent behavioral factors: 1) attention deficits, 2) lack of emotional stability, 3) poor social skills, 4) poor physical appearance, 5) special physical problems, 6) special behavioral problems, and 7) poor school attendance. Marks for all subjects were recorded for both semesters of the school year by a school official using local school records and coded numerically (5). Because of the range of ages, the children were not enrolled in all academic subjects simultaneously. Five major academic subjects were tested: reading (including comprehension), language arts (including oral recitation, composition, grammar, English, discussion, and spelling), mathematics, general science, and social science (social education and social studies).

Socioeconomic and home environmental conditions. We have reported on details concerning a questionnaire administered to the primary caretaker by a community health sister who was generally familiar with the home (11). Briefly, items on the questionnaire were classified by a principal components factor analysis with normal varimax rotation. This analysis grouped related items into nine independent factors, including: 1 ) socioeconomic characteristics of the home, 2) adult interaction with the child and amount of stimulation in the home, 3) level of maternal education, 4) number of caretakers, 5) type of transportation used, 6) type of and hours of mother's work, and 7) presence of the father or adult males in the home.

Data analysis. All analyses were performed using the Statistical Package for the Social Sciences (12). Eleven-plus scores were compared using a two-way ANOVA ( $\operatorname{sex} \times$ nutritional history), supplemented by a Student Newman-Keuls test to determine which groups differed when a significant nutritional history effect was found. Pearson correlations were used to correlate 11-plus scores with IQ scores, factors scores describing classroom behavior and marks in school obtained when the children were 5- to 11-y-old, and also with factor scores describing socioeconomic characteristics of the households. MRA was used to calculate 11plus scores corrected for socioeconomic factors (residual scores).

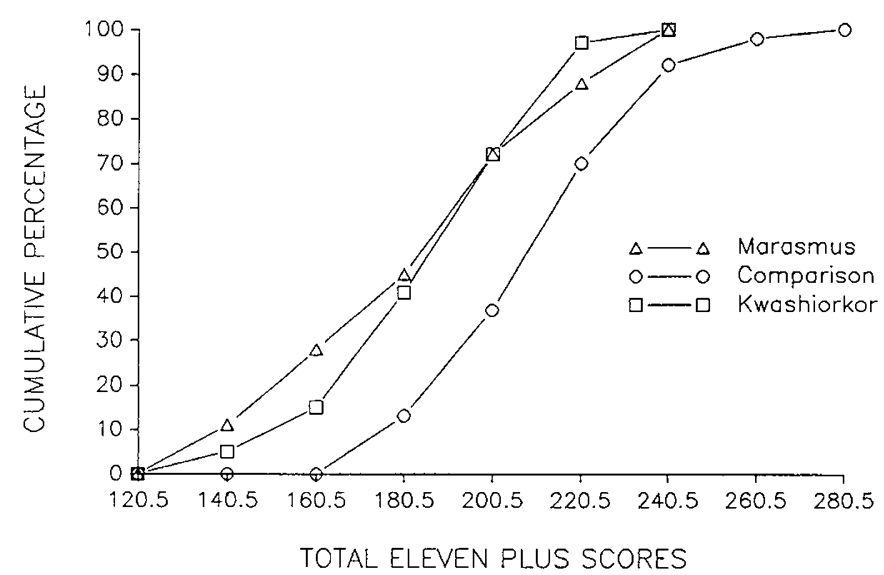

Fig. 1. Cumulative distribution of 11-plus scores in children with histories of marasmus $(n=64)$ or kwashiorkor $(n=39)$ and comparison children $(n=63)$.

Table 1. Mean 11-plus examination scores $\pm S D$ of children with histories of malnutrition and comparison children*

\begin{tabular}{lcc}
\hline & Female & Male \\
\hline Comparison & $222.08 \pm 23.63 \dagger$ & $202.67 \pm 18.01 \dagger$ \\
$n$ & 24 & 39 \\
Marasmus & $188.00 \pm 25.65$ & $178.28 \pm 30.03$ \\
$n$ & 25 & 39 \\
Kwashiorkor & $182.40 \pm 18.15$ & $186.79 \pm 25.42$ \\
$n$ & 15 & 24 \\
Total & $199.47 \pm 29.05$ & $189.61 \pm 26.94$ \\
$n$ & 64 & 102 \\
\hline
\end{tabular}

*ANOVA shows significant effects of $\operatorname{sex}[F(1,160)=6.74 ; p<$ $0.01]$ and nutritional group $[F(2,160)=24.12 ; p<0.001]$, but no interaction.

$\dagger$ The comparison group differs significantly from the other two groups (Student Newman-Keuls test; $p<0.05$ ). 


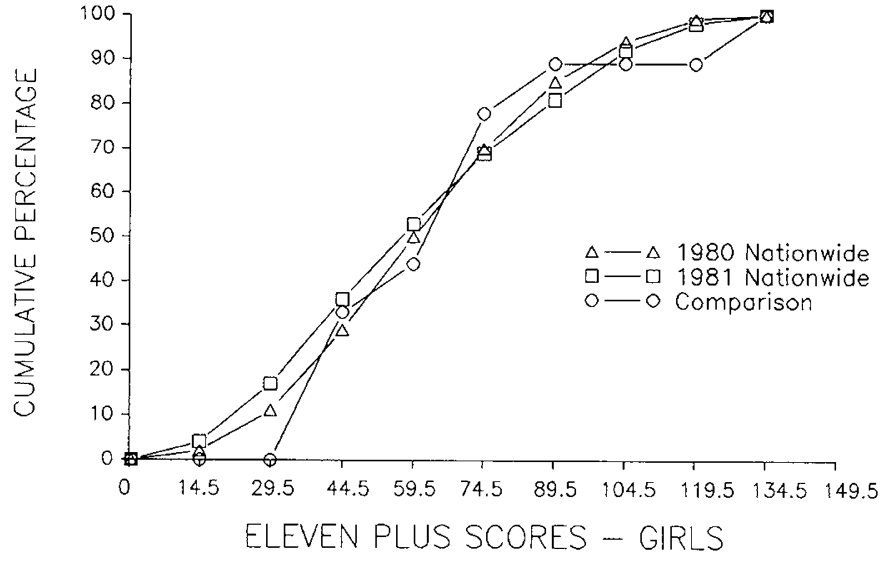

Fig. 2. Cumulative distribution of English 11-plus raw scores of the comparison girls in relation to national scores on the 1980 and 1981 examinations.

Table 2. Correlation coefficients (n) between 11-plus scores at

11 y of age and IQ, classroom behavior factors, and school performance at 5 to 11 y of age

\begin{tabular}{lcc}
\hline \multicolumn{1}{c}{ Independent variables } & Comparison & Marasmus \\
\hline Full scale IQ & $0.61^{*}(63)$ & $0.69^{*}(63)$ \\
& & \\
Classroom behavior factors: & & \\
Attentional deficits & $0.46^{*}(63)$ & $-0.60^{*}(64)$ \\
Poor emotional stability & 0.13 & 0.09 \\
Poor social skills & -0.03 & 0.21 \\
Poor physical appearance & 0.11 & 0.15 \\
Special physical problems & 0.06 & 0.00 \\
Special behavior problems & 0.14 & -0.15 \\
Poor attendance & 0.09 & 0.12 \\
& & \\
Marks in: & & \\
Reading & $0.54^{*}(32)$ & $0.71^{*}(25)$ \\
Language arts & $0.47^{*}(46)$ & $0.66^{*}(46)$ \\
Mathematics & $0.31 \dagger(45)$ & $0.56^{*}(47)$ \\
General science & $0.30(30)$ & $0.60^{*}(31)$ \\
Social science & $0.30(30)$ & $0.71^{*}(31)$ \\
\hline
\end{tabular}

$* p<0.001$.

$\dagger p<0.05$.

Table 3. Pearson correlations between 11-plus examination scores and home environmental factors for previously malnourished and comparison children $(n=127)$

\begin{tabular}{lc}
\hline Independent variables & Correlation $(r)$ \\
\hline Family stability & $0.29^{*}$ \\
Adult-child interaction & $0.32^{*}$ \\
Home conveniences & $0.32^{*}$ \\
Mother's education & $0.24 \dagger$ \\
Transportation & 0.13 \\
Mother's occupation & -0.00 \\
Adult males in the home & 0.01 \\
\hline
\end{tabular}

$* p<0.001$

$+p<0.01$.

\section{RESULTS}

Eleven-plus scores in relation to nutritional history and sex. The distribution of total standardized scores on the 11-plus examination in children with histories of marasmus or kwashiorkor and in comparison children is illustrated in Figure 1. This figure demonstrates a disparate number of cases at the lower end of the distribution curve for the two previously malnourished groups. Values for kwashiorkor and marasmus cases showed the same shift to the left of comparison values because of lower scores in these two groups of children.
Mean 11-plus scores and SD, classified by sex and nutritional history, are reported in Table 1. Statistical comparisons of these values were made using a two-way ANOVA with sex and nutritional history as independent variables and the 11-plus score as the dependent variable (Table 1). This test demonstrated significant effects of both sex and nutritional history. Girls had significantly higher scores than boys, with an advantage of approximately 10 points for all three groups of girls as compared with boys. Student Newman-Keuls tests showed that comparison children scored higher than children with histories of marasmus or kwashiorkor $(p<0.05)$. However, scores achieved by children with histories of marasmus or kwashiorkor were not different. There was no significant interaction between sex and nutritional history.

In a supplementary analysis, we addressed the possibility that there were variations in scores attributable to the child's age (because of different examinations having been administered each year). When we corrected the ANOVA for the child's age in 1984, we found no significant effect of age. Therefore, we have not included this variable in any subsequent analyses described in the text

Finally, we compared 11 -plus scores achieved by the comparison children with national scores during the same period. This comparison was made using raw scores, calculated separately for the English and math tests. As shown in Figure 2, for the combined results of the 1980 and 1981 English examinations, scores achieved by comparison children in the current study had a similar distribution to scores achieved by the total population of children tested during the same period. In general, higher scores were obtained by girls as compared with boys on the nationwide examination, as was the case for children included in our study. Similar patterns were present for boys and girls, and also for math scores, which are not reported here.

In summary, low 11-plus scores were correlated with an early history of kwashiorkor or marasmus. Values achieved by comparison children in this study were consistent with national norms, whereas scores of previously malnourished children were substandard.

Eleven-plus scores in relation to $I Q$, academic performance, and classroom behavior at 5 to 11 y of age. Because the children in our study had been followed from early childhood, it was possible to determine the extent to which 11-plus scores were associated with school and cognitive performance at earlier ages. Pearson correlations were used to quantify the degree of association between the 11-plus scores at $11 \mathrm{y}$ of age and IQ, classroom behavior, and academic performance at 5 to $11 \mathrm{y}$ of age. These analyses were restricted to children with histories of marasmus and comparison children for whom these earlier data were available.

As may be seen in Table 2, there were a number of moderately to highly significant correlations between the 11-plus scores and IQ performance, classroom behavior, and academic performance for previously malnourished and comparison children in the study. When classified by nutritional group, a similar degree of association was found between 11-plus scores and IQ and classroom behavior in both previously malnourished and comparison children. These relationships were identical even after controlling for age, and they were similar for boys and girls. However, academic performance, especially social science grades, was more strongly associated with 11-plus scores among the previously malnourished children $(z=2.14)$. The moderate degree of correlation between 11-plus scores and academic grades can be attributed to the lengthy interval between the two measures (up to $7 \mathrm{y}$ ).

Next, we determined whether IQ scores and classroom behavior at younger ages were associated with the 11-plus scores to the same degree as performance at older ages when the child was closer in age to the age when the 11-plus was administered. This was done by calculating Pearson correlations between the 11plus scores and IQ and classroom behavior at 5 to $8 \mathrm{y}$ of age and 
Table 4. Correlations between 11-plus scores and independent variables and part correlations controlling for nutritional history, IQ, classroom behavior and home environment*

\begin{tabular}{|c|c|c|c|c|c|c|}
\hline \multirow[b]{2}{*}{$\begin{array}{l}\text { Independent } \\
\text { variables }\end{array}$} & \multirow[b]{2}{*}{$\begin{array}{c}\text { Simple } \\
\text { correlations } \\
(r)\end{array}$} & \multicolumn{5}{|c|}{$\begin{array}{l}\text { Part correlations } \\
\text { (part } r \text { ) controlling for }\end{array}$} \\
\hline & & $\begin{array}{l}\text { Nutritional } \\
\text { history }\end{array}$ & IQ & $\begin{array}{c}\text { Classroom } \\
\text { behavior }\end{array}$ & $\begin{array}{c}\text { Home } \\
\text { environment }\end{array}$ & $\begin{array}{c}\text { All } \\
\text { variables } \\
\text { (total part } r \text { ) }\end{array}$ \\
\hline Nutritional history & $0.48 \dagger$ & & $0.19 \neq$ & $0.12 \S$ & $0.20 \ddagger$ & -0.02 \\
\hline IQ & $0.73 \dagger$ & $0.58 \dagger$ & & $0.37 \dagger$ & $0.54 \dagger$ & $0.32 \dagger$ \\
\hline Classroom behavior & $0.75 \dagger$ & $0.60 \dagger$ & $0.42 \dagger$ & & $0.54 \dagger$ & $0.32 \dagger$ \\
\hline Home environment & $0.59 \dagger$ & $0.41 \dagger$ & $0.34 \dagger$ & $0.28 \ddagger$ & & $0.22 \ddagger$ \\
\hline
\end{tabular}

* Pearson $r$ for nutrition group and IQ; multiple $R$ for classroom behavior and home environment; part $r$ were calculated by hierarchial MRA in which control variables were entered before the independent variables.

$$
\begin{aligned}
& \dagger p<0.001 . \\
& \ddagger p<0.01 . \\
& \S p<0.05 .
\end{aligned}
$$

at 9 to $11 \mathrm{y}$ of age. (Academic performance could not be used because of the small sample size.) Correlations between IQ and 11-plus performance were significant to a similar degree for both younger $(r=0.64 ; p<0.001 ; n=53)$ and older children $(r=$ $0.77 ; p<0.001 ; n=73$ ). Classroom behavior was also significantly correlated with 11-plus scores in the younger and older age groups ( $r=0.73$ and $0.78, p<0.001$, respectively).

These data suggest that both IQ and classroom behavior applied in the early school years can predict impaired performance on the 11-plus examination at $11 \mathrm{y}$ of age; this finding has important implications for developing intervention strategies.

Socioeconomic and home conditions. These findings were examined in relationship to concurrent socioeconomic and home environmental conditions when the children were 5 to $11 \mathrm{y}$ of age. We have previously reported that homes of previously malnourished children were disadvantaged as compared with those of well-nourished children despite careful attempts to control for this in the selection of the sample (10). We also reported $(1,2)$ the home environmental conditions both at the time of the episode and during adolescence were similar in the marasmus and kwashiorkor groups.

The correlations $(r)$ between the individual socioeconomic and home environmental factors and the 11-plus scores are shown in Table 3. Four environmental variables were significantly correlated with 11-plus performance. In order of importance, those factors that retained a positive relationship with the 11-plus scores were the quality of parent-child interaction, number of caretakers, home conveniences present in the household, and level of maternal education. We therefore repeated the comparison between 11-plus scores of previously malnourished and comparison children while controlling for the home environmental factors. We used a MRA to calculate 11-plus scores corrected for the effects of the environmental factors (residual 11 -plus scores). ANOVA applied to the residual scores confirmed that 11 -plus scores achieved by children with previous malnutrition were significantly lower than scores of comparison children. There were also significant sex effects $[F(1,123)=6.0 ; p<0.5]$ because of higher scores for the girls. Correcting for socioeconomic and home conditions when the children were 11 to $18 \mathrm{y}$ of age (2) yielded similar results with respect to nutritional group but no sex effects.

In summary, these results confirm that home environment was associated with 11-plus performance. Nevertheless, an early history of malnutrition (and related conditions in the first year of life) was correlated with 11-plus performance at $11 \mathrm{y}$ of age independent of home environmental conditions.

Relationships between all factors. Table 4 summarizes the combined and independent contribution of the history of malnutrition, sex, IQ, group of classroom behaviors (seven factors) and group of home environmental factors (seven factors) to 11 plus performance. These relationships were tested using Pearson tests (simple $r$ ) to correlate 11-plus scores with nutritional history and IQ and multiple $r$ were used to correlate 11-plus scores with the group of classroom behavior factors and home environment factors. Part correlations (part $r$ ) were calculated by applying hierarchical MRA in which the control variables were entered first and the independent variable entered last. The multiple correlation $(r)$ between all 17 predictors with 11-plus performance was 0.876 , accounting for $76 \%$ of the variance in 11 -plus scores.

As seen in the 1st column of Table 4, each set of measures was significantly correlated with the 11-plus scores (Pearson $r$ ). The most striking associations were present in the case of IQ $(p<$ $0.001)$ and the group of classroom behaviors $(p<0.001)$, which were both significantly correlated with 11-plus scores even when all other variables were controlled for (total part $r$ appearing in the final column). The correlation between a previous history of malnutrition variable and 11-plus scores $(r=0.48)$ accounted for $23 \%$ of the variance (simple correlation). However, the part correlation between the history of malnutrition and 11-plus performance $(r=0.12)$ accounted for only $1 \%$ of the variance when classroom behaviors were controlled for (part $r$ controlling for classroom behavior) and was not significant when all other variables were controlled (total part $r$ ). This suggests that the effects of malnutrition on 11-plus performance are indirect and mediated predominantly by deficits in classroom behavior.

In addition, factors in the home environment played a significant but moderate role in 11-plus performance, even after all other variables were controlled (total part $r$ ). Controlling for the home environmental factors reduced but did not eliminate the relationships between the other measures tested and 11-plus performance (part $r$ controlling for home environment).

In summary, these findings underscore the stability of relationships between behavioral and cognitive factors measured at 5 to $11 \mathrm{y}$ of age and 11 -plus performance at $11 \mathrm{y}$. They also suggest that the primary mechanism whereby a history of malnutrition affects 11 -plus scores is indirect and mediated predominantly by deficits in classroom behavior (especially attention deficits), followed by independent effects of IQ and conditions in the home environment.

\section{DISCUSSION}

We have previously reviewed the literature relating to school performance and early childhood malnutrition (6). Briefly, in a series of 20 South African children with histories of infantile marasmus and 20 matched controls followed up to $23 \mathrm{y}$ of age, Stoch et al. (13) reported fewer years of schooling and lower school performance, especially in girls, after the history of marasmus. Richardson (14), in his series of 75 Jamaican schoolboys, reported that boys with histories of malnutrition and their siblings had lower scores on the Wide Range Achievement Test at 
6 to $10 \mathrm{y}$ of age and had lower marks in school than their siblings or classmates at these ages. In our earlier studies of Barbadian school children, we have reported that educational performance measured by marks in school (5) and classroom behavior (10) is inferior among boys and girls with histories of childhood malnutrition. However, malnutrition secondary to organic illness was generally not found to be associated with impaired classroom performance (6), except by Klein et al. (15) in Canada.

Published studies of the 11-plus examination are limited to a Western (British) population. In a study of school children from Birmingham, Barker and Edwards (16) reported on the relation between obstetric history and performance on the 11-plus examination. Record et al. (17) demonstrated the combined influence of maternal age, birth order, and social class on 11-plus scores; they also found small but significant sex effects on examination scores. Finally, Hebel et al. (18) found that 11-plus scores in Birmingham were not significantly correlated with levels of lead pollution in the local community.

In our study, we showed that a history of malnutrition and related events in the first year of life were associated with impaired performance on the national 11-plus examination administered at $11 \mathrm{y}$ of age. Scores of children with histories of marasmus or kwashiorkor in the first year of life were significantly reduced as compared with those of control children who had no histories of malnutrition. There were no differences attributable to the type of infantile malnutrition. The lack of educational progress and low marks on the common entrance examination in the index cases were closely associated with a range of intellectual and behavioral deficits previously reported in this group of children in IQ scores (9), classroom behavior (10), and academic performance on all major academic subjects (5). When we controlled for each of these factors (Table 4), we found that poor performance on the high school examination was almost completely attributable to behavioral deficits in the classroom and specifically to attentional problems, with a smaller but significant contribution from deficits in IQ.

Poor performance on the 11-plus examination was strongly associated with behavioral and cognitive deficits measured as early as 5 to $8 \mathrm{y}$ of age. This suggests that classroom observations and intellectual testing in the early school years can be applied to predict future impaired performance on the 11-plus examination at $11 \mathrm{y}$ of age. This is particularly important because of the possibility of establishing successful interventions in the early school years. Interventions focused on younger school children may serve to improve subsequent 11-plus performance and provide the opportunity for greater achievement in the local community.

Finally, careful analysis of the home background of children in the study led us to conclude that, even under the relatively uniform conditions in Barbados and the careful selection and matching procedures in this study, there were some differences between groups of previously malnourished children and the comparison children. However, factors in the home environment of the children at ages 5 to $11 \mathrm{y}$ did not completely explain the effects of early malnutrition on performance on the national examination (Table 4). Thus, the correlation between a history of malnutrition and performance on the national high school examination continued to be significant even when corrected for the effects of home environmental conditions. The relationship between 11 -plus scores and IQ and classroom behavior was also unchanged even after correction for environmental conditions (Table 4). This does not preclude a more prominent role of environmental factors during early childhood when these may have contributed to or exacerbated the episode of malnutrition. Environmental deprivation at these earlier ages may also serve as an independent cause of attention deficit disorder in children (19).

These findings have important implications for the futures of children with histories of early malnutrition. This event and any related conditions early in life may restrict educational opportunities, and thereby impact life-long adjustment.

Acknowledgments. The authors thank Marjorie Bowen and Jean Ramsey, community health sisters, for their assistance in collecting the data, and Dr. Robert Harrison for statistical advice.

\section{REFERENCES}

1. Galler JR, Ramsey F, Salt P, Archer E 1987 The long term effects of early kwashiorkor compared with marasmus. I. Physical growth and sexual maturation. J Pediatr Gastroenterol Nutr 6:841-846

2. Galler JR, Ramsey F, Salt P, Forde V 1987 The long term effects of early kwashiorkor compared with marasmus. II. Intellectual performance. $\mathrm{J} \mathrm{Pe}-$ diatr Gastroenterol Nutr 6:847-854

3. Galler JR, Ramsey F, Salt P, Archer E 1987 The long term effects of early kwashiorkor compared with marasmus. III. Fine motor skills. J Pediatr Gastroenterol Nutr 6:855-859

4. Galler JR, Ramsey F, Archer E 1989 A follow-up study of the influence of early malnutrition on subsequent development. VI. Home and classroom behavior. J Am Acad Child Adolesc Psychiatry 28:254-261

5. Galler JR, Ramsey F, Solimano G 1984 The influence of early malnutrition on subsequent behavioral development. III. Learning disabilities as a sequel to malnutrition. Pediatr Res 18:309-313

6. Galler JR 1984 The behavioral consequences of malnutrition in early life. In: Galler JR (ed) Nutrition and Behavior. Plenum Press, New York, pp 63117

7. Ramsey FC 1982 Protein-Energy Malnutrition in Barbados. The Role of Continuity of Care in Management. Josiah Macy Jr Foundation, New York

8. Gomez F, Galvan RG, Cravioto J, Frank S 1954 Malnutrition in infancy and childhood, with special reference to kwashiorkor. Adv Pediatr 7:131-169

9. Galler JR, Ramsey F, Solimano G, Lowell WE 1983 The influence of early malnutrition on subsequent behavioral development. I. Degree of impairment in intellectual performance. J Am Acad Child Adolesc Psychiatry 22:815

10. Galler JR, Ramsey F, Solimano G, Lowell WE 1983 The influence of early malnutrition on subsequent behavioral development. II. Classroom behavior. $J$ Am Acad Child Adolesc Psychiatry 22:16-22.

11. Galler JR, Ramsey F 1985 The influence of early malnutrition on subsequent behavioral development. VI. The role of the microenvironment of the household. Nutr Behav 2:161-173

12. SPSS Inc. 1988 SPSS-X User's Guide, 3rd ed. McGraw-Hill, New York

13. Stoch MB, Smythe TM, Moodie AD, Bradshaw D 1982 Psychosocial outcome and CT findings after growth under nourishment during infancy: a twentyyear developmental study. Dev Med Child Neurol 24:419-436

14. Richardson SA 1980 The long range consequences of malnutrition in infancy: a study of children in Jamaica, West Indies. In: Wharton B (ed) Topics in Pediatrics. Vol 2, Nutrition in Childhood. Pitman, London 163-176

15. Klein PS, Forbes GB, Nadar PR 1975 Effects of starvation in infancy (pyloric stenosis) on subsequent learning abilities. J Pediatr 87(1):8-15

16. Barker DJP, Edwards JH 1967 Obstetric complications and school performance. Br Med J [Clin Red] 3:695-699

17. Record RG, McKeown T, Edwards JH 1969 The relation of measured intelligence to birth order and maternal age. Ann Hum Genet 33:61-79

18. Hebel JR, Kinch D, Armstrong T 1976 Mental capability of children exposed to lead pollution. Br J Prev Soc Med 30:170-174

19. American Psychiatric Association 1987 Diagnostic and Statistical Manual of Mental Disorders, 3rd ed, revised. American Psychiatric Association, Washington, DC 